\title{
Reliable, Efficient, and Power Optimized Control-Channel Selection Scheme for Cognitive Radio Networks
}

\author{
Saleem Aslam, Adnan Shahid, and Kyung Geun Lee \\ Department of Information and Communication Engineering, Sejong University, Seoul 143-747, Republic of Korea \\ Correspondence should be addressed to Kyung Geun Lee; kglee@sejong.ac.kr
}

Received 12 July 2013; Revised 18 October 2013; Accepted 24 October 2013

Academic Editor: Xi-Ming Sun

Copyright ( 2013 Saleem Aslam et al. This is an open access article distributed under the Creative Commons Attribution License, which permits unrestricted use, distribution, and reproduction in any medium, provided the original work is properly cited.

\begin{abstract}
This paper presents a centralized control-channel selection scheme for cognitive radio networks (CRNs) by exploiting the variation in the spectrum across capacity, occupancy, and error rate. We address the fundamental challenges in the design of the controlchannel for CRNs: (1) random licensed users (LUs) activity and (2) the economical and vulnerability concerns for a dedicated control-channel. We develop a knapsack problem (KP) based reliable, efficient, and power optimized (REPO) control-channel selection scheme with an optimal data rate, bit error rate (BER), and idle time. Moreover, we introduce the concept of the backup channels in the context of control-channel selection, which assists the CRs to quickly move on to the next stable channel in order to cater for the sudden appearance of LUs. Based on the KP and its dynamic programming solution, simulation results show that the proposed scheme is highly adaptable and resilient to random LU activity. The REPO scheme reduces collisions with the LUs, minimizes the alternate channel selection time, and reduces the bit error rate (BER). Moreover, it reduces the power consumed during channel switching and provides a performance, that is, competitive with those schemes that are using a static control-channel for the management of control traffic in CRNs.
\end{abstract}

\section{Introduction}

In recent years, most researchers and leading communication agencies have decided to adopt the dynamic spectrum management framework for handling the spectrum requirements of upcoming advanced communication systems. Such a shift in the spectrum management paradigm requires a robust communication radio that can meet the stringent demands of the future generation of communication systems. Cognitive radio (CR), or reconfigurable radio, seems to be the best technology for dynamic spectrum access [1-4]. CRs employ the spectrum-sensing scheme to locate empty spots within the radio spectrum band and then utilize the software routines using software-defined radio (SDR) for parameter adjustment. The CR can operate in the vacant portion of the licensed band only if they operate under a tolerable interference limit with LUs [5]. From the perspective of the CR, there are five main functions spectrum sensing: spectrum analysis, spectrum decision, spectrum sharing, and spectrum mobility [2-5]. As an example, the network control systems (NCS) are heavily investigated to manage the packet drop and latency of control-channel $[6,7]$. Various methods for evaluating the stability analysis of the NCS are discussed in [8-10]. However, these schemes are focused for NCS rather than the dynamic communication environment like CRNs.

To locate and select the best control-channels in terms of availability time and quality, spectrum sensing, analysis, and decision schemes play a crucial role. The spectrum sharing scheme helps in the optimal allocation of the available free channel/spectrum among competing CRs, whereas the spectrum mobility helps the CRs to avoid interference with the LUs by switching the CR to the next available channel. From the perspective of the LU networks, a user has the legitimate right to use the spectrum under the contract of the service provider. To have better coordination and synchronization among different components, the CRs need to exchange significant amount of the control information to make a timely decision about spectrum sensing, sharing, and mobility tasks [11]. Therefore, a stable and better quality control-channel is required to meet the desired efficiency. By selecting a better quality control-channel, the CRNs can provide a better communication environment to its users and 
can manage the control tasks in an appropriate way to support a wide range of applications including internet chat, voice calls, and live video streaming.

A predefined control-channel can be utilized for the management of channel selection and channel allocation tasks [12-17]. However, channel sensing and selection tasks in CRNs depend directly on the relative distance of the CR from the LU. Therefore, the selection of a predefined single channel may experience collisions with LUs. A CORVUS system given in [13] introduces a method for the management of the control information in CRNs. The given scheme employs a dedicated control-channel within the ultrawide band (UWB) to manage the control traffic of CRNs. Similarly, several other schemes [14-17] exploit the UWB based dedicated controlchannel for CRNs. Besides the UWB, many authors introduce the concept of utilizing the industrial scientific and medical (ISM) band for the management of the control tasks $[12,18$, 19]. Although these schemes provide simpler solutions, these schemes are struggling with interference from other devices and spectrum availability issues. In [20], the authors describe an orthogonal frequency division multiplexing (OFDM) based wide-range transmission of control messages with small bit error rates. However, the scope of such schemes is very limited.

Although a dedicated control-channel seems to be a simpler solution, it inherently has two fundamental challenges: (1) the random behavior of the LUs and (2) additional cost and vulnerability concerns. The LUs have legitimate rights to use the spectrum band. Consider a channel where CRs are exchanging control traffic, after a certain interval the LU arrives on that particular channel. In such a situation, the CRs should vacate the current channel and tune them to a new control-channel to avoid possible interference. To detect the LUs arrival activity, many spectrum-sensing schemes are proposed in the literature. The collaborative spectrum-sensing schemes [21-23] combine the observation of individual CRs to make a final decision regarding the presence or absence of an LU over a particular channel. Thus, it is extremely important for the control-channel design scheme to have an accurate estimation regarding the activity of the LUs across the spectrum and time. Secondly, dedicating the control-channel within the ISM band increases the implementation cost of the CRNs, which is also against the fundamental principal of the adaptive CR. Moreover, there are also vulnerability concerns in using the ISM band. The use of unlicensed bands cannot guarantee the reliability of control traffic, which is especially crucial for CRN operation. Another important factor for CRNs is the spectrum heterogeneity. Each CR finds a different set of available channels based upon its geographic location. Due to the heterogeneous nature of the spectrum, it is difficult to locate a common controlchannel at a particular time.

In spite of the inherent issues and limitations of dedicated control-channels, there are few research efforts in the area of dynamic control-channel design [24-28]. The dynamic model of the control-channel can counter the limitations of the dedicated model by selecting the control-channels in the light of LU dynamics and the varying nature of the radio spectrum. Zhao et al. propose a distributed coordination of CRs via a locally selected control-channel that may vary dynamically in response to the LU's arrival [26]. The CRN is grouped into different numbers of clusters based on the number of common channels. This scheme can reduce the distinct frequency bands used as control-channels (equal to number of clusters). However, if the LU arrives frequently, the scheme needs to perform reclustering again and again which can degrade the QoS of the CRNs. Similar cluster oriented schemes are also discussed in [27, 28]. However, these schemes cannot provide the mechanism to handle the interference and alternate channel search time. Moreover, these schemes do not provide details regarding the exchange of control information at the intralevel (controlled by a single base station).

In $[29,30]$, the authors introduce sequence based controlchannel selection schemes. In such schemes, the CRs tune them to sensed channels on a predefined or pseudorandom sequence until they meet a common channel. The information is exchanged through broadcast messages which contain the list of available channels. Having information from a neighbor, a CR updates its list of common control-channels and hop on to channels on a sequence that may differ from its neighbor. As soon as the CRs exchange the synchronization frames among each other, they agree on a common hopping sequence for data exchange. Although the given schemes provide a usable framework, they have two prime limitations. First, if the CR broadcasts the list of channels without LU effects, it leads to a major question mark on their practical application. Secondly, there is significant time consumption during synchronization, which limits the actual use of the spectrum. In the synopsis, all previously mentioned schemes are proposed on the implicit requirements and assumptions that might limit their applicability to the job of dynamic control-channel assignment.

In this paper, we introduce a knapsack problem (KP) based control-channel selection scheme for centralized CRNs and employ a dynamic-programming approach to get the optimal solution of KP. We dynamically select the controlchannels over time and manage the random appearance of LUs over different channels by exploiting their prior statistics. The main contributions of the paper are as follows.

(1) We formulate the control-channel selection problem as an optimization problem with the objective of maximizing the capacity and the mean idle time of channels under the constraints of data rate and error rate. The quality of an item in KP is represented using capacity, error rate, and mean idle time and the KP's capacity is considered to be three (one main and two backup channels).

(2) We develop a model to compute the mean idle time of the channels that predicts availability of the channel to CRs. The busy-state shows the presence of the LU and idle-state indicates the availability of the given channel to CRs for the transmission of their control traffic.

(3) We utilize the concept of backup channels inside the domain of the control-channel selection of CRNs. The backup channels assist the CRs to complete the 
on-going control data transmission, and it reduces the alternate channel search time. We present a mathematical relationship to compute the hand-off delay using two backup control-channels.

(4) Finally, we evaluate the performance of the REPO scheme with the existing schemes across different important factors such as collision with LUs, hand-off delay, power, and reliability (least BER).

The rest of the paper is organized as follows. Section 2 describes the system model and proposed framework for REPO scheme. Section 3 covers the mathematical formulation of the problem. Section 4 shows the experimental setup and simulation results. Finally, Section 5 concludes with the findings of the paper.

\section{System Model}

We consider a centralized CRN, where the LUs and CRs are using the spectrum in the same geographical area. The LUs have legitimate rights to use the spectrum according to terms and condition of their service providers. However, the users of CRNs can use the spectrum of the LUs as long as they operate under the tolerable interference limit. The spectrum of the LU can be grouped into a set of $M=\left\{m_{1}, m_{2}, \ldots, m_{p}\right\}$ channels. Each channel $m_{k}$ has a unique data capacity, BER and LU activity index, or idle time. There are total $N=\left\{n_{1}, n_{2}, \ldots, n_{q}\right\}$ intra-CRNs and $L=\left\{l_{1}, l_{2}, \ldots, l_{r}\right\}$ indicates the number of CRs in different intra-CRNs. Each CR performs spectrum-sensing task and forwards its report to the respective CR base station. Later on, the base station forwards the sensing reports to the central control-channel manager (CCM). The CCM is the main entity that applies a KP based REPO scheme for the selection of the controlchannel for the management of the control tasks. For accurate spectrum sensing, we consider that CRs are equipped with cyclostationary feature detection based cognitive sensors. Whenever the LU arrives on the current selected controlchannel, the CR immediately vacates the channel and informs the CRBS. Later on, the CRBS reallocates other channels for control tasks after collaboration with CCM.

2.1. Nomenclature. The main notations used in the rest of the paper are as follows.

$$
\begin{aligned}
& N \text { : Set of cells or intra CRNs } \\
& M \text { : Set of channels } \\
& L: \text { Set of CR } \\
& p: \text { Total number of channels } \\
& q: \text { Total number of cells or intra CRNs } \\
& r: \text { CRs per cell } \\
& l: \text { Total number of selected control-channel } \\
& \beta_{1}: \text { Weight of data rate objective function } \\
& \beta_{2}: \text { Weight of idle time objective function } \\
& \beta_{3}: \text { Weight of BER objective function }
\end{aligned}
$$

$C_{k}$ : Data rate on $k$ th channel

$\gamma_{k}$ : Mean idle time on $k$ th channel

$\sigma_{j}$ : A sensing vector of CR

$\xi_{j}^{i}$ : Sensing reports from a local base station

$\eta_{k}$ : Interference plus noise contents of $k$ th channel.

The main assumptions of the REPO scheme are as follows.

(i) In order to have a common control-channel for initialization of the CRN, the CCM employs a global channel (GC) of bandwidth $B \mathrm{~Hz}$ in the ISM band to discover the network topology and exchange relevant initial information such as spectrum availability. Once the initial connection is established, the CCM apply REPO scheme for selection of the best quality control-channel.

(ii) We assume that CRs are equipped with a single transceiver and CRs can perform sensing and data transmission tasks based on the designated time for each mode.

(iii) In every intra-CRN, a CR performs the LU detection task on a regular basis and reports the sensing result to the respective CRBS. Moreover, we assume that the CCM is perfectly synchronized with other CRBSs to collect the sensing results and that CRBSs can communicate directly with the CCM.

(iv) The sensing task is repeated after each application round. An application round represents the validity of the current channel selection in terms of the number of time slots.

(v) A CRBS with the maximum number of active CRs can act as CCM.

2.2. The Proposed Framework. This section describes the working of the proposed system. The main tasks involved are (1) LUs presence detection, (2) mean idle time prediction, and (3) quality estimation as shown in Figure 1.

2.2.1. LUs Presence Detection (LPD). One of the primary tasks in the CRNs is to detect the activity of the LU. It helps to minimize the interference from possible collisions of CRs with LUs. The sensing operation of the CR can be described using the binary hypothesis mentioned in [23] using

$$
\begin{gathered}
H_{0}, \quad a_{k}=I_{k}, \\
H_{1}, \quad a_{k}=a_{k} \cdot h_{k}+I_{k},
\end{gathered}
$$

where $k$ represents the channel index within the radio spectrum and $H_{0}$ corresponds to a hypothesis, when the $\mathrm{LU}$ is absent and only the noise signal exists over the $k$ th channel. The hypothesis $H_{1}$ represents a case in which the LU is present over the channels with slight contents of noise. We adopt the cyclostationary feature detection scheme to detect the presence of LU over different channels. A signal $s(t)$ is considered to be second order cyclostationary (in the wide 


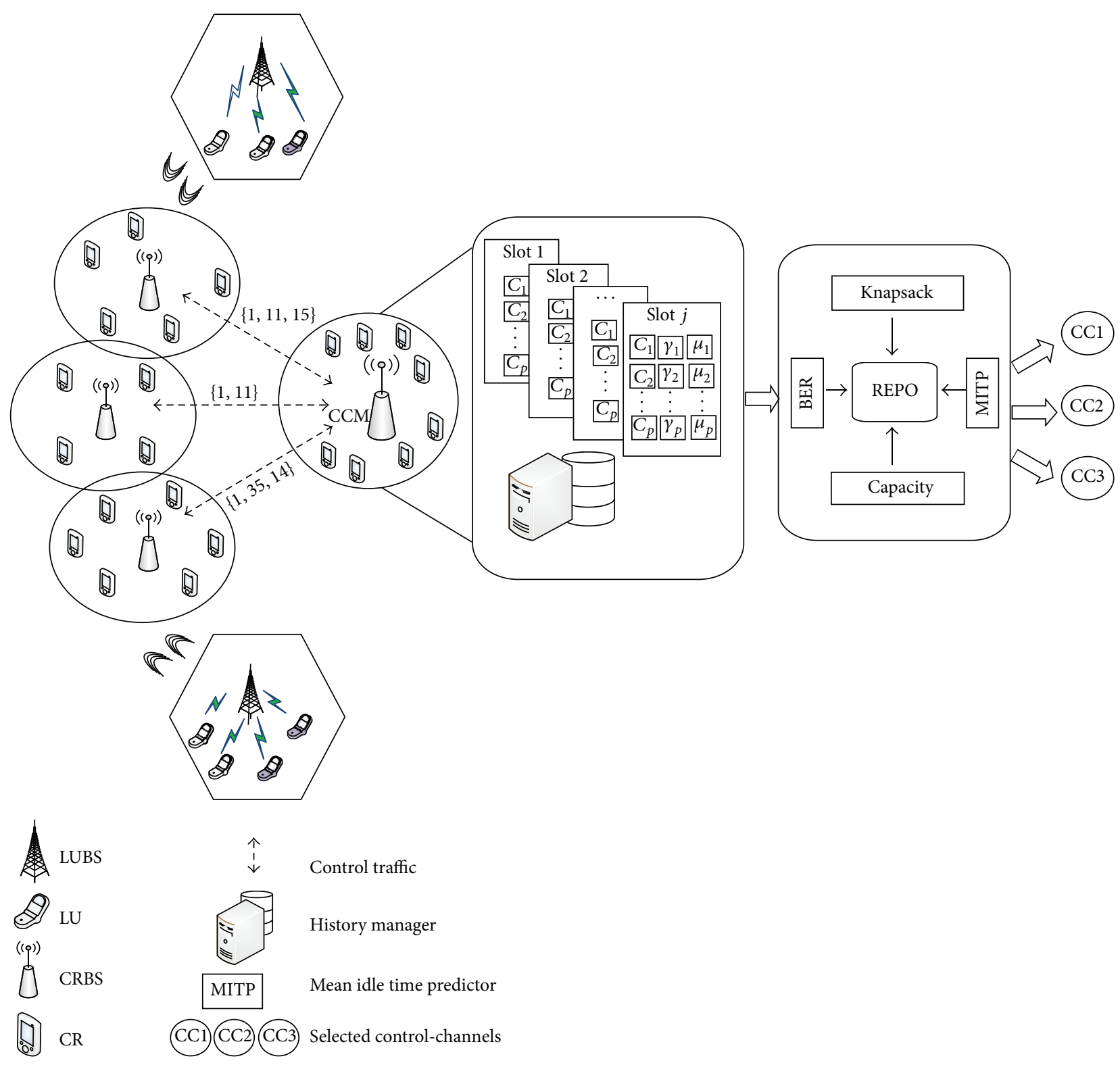

FIgURE 1: Proposed framework of REPO scheme.

sense) if its autocorrelation function can be represented by the expression

$$
R_{s}(t, \tau)=E\left\{s\left(t+\frac{\tau}{2}\right) s\left(t-\frac{\tau}{2}\right)\right\}
$$

where the signal $R_{s}(t, \tau)$ is periodic and its characteristics can be measured using the autocorrelation function given in [31]:

$$
R_{s}^{\alpha}(\tau)=\lim _{\Delta t \rightarrow \infty} \frac{1}{\Delta t} \int_{-\Delta t / 2}^{\Delta t / 2} s\left(t+\frac{\tau}{2}\right) \cdot s\left(t-\frac{\tau}{2}\right) e^{-i 2 \lambda \alpha t} d t
$$

where $\alpha$ represents the cyclic frequency and measured with interval $\Delta t$. The second order cyclostationary signals provides discrete correlation patterns which occur in the signal $s(t)$.
The patterns can be expressed using the spectral correlation function [17] and used for the analysis of LUs presence [32]:

$$
\begin{gathered}
S_{s}^{\alpha}(f)=\lim _{\Delta t \rightarrow \infty} \lim _{\Delta f \rightarrow \infty} \frac{1}{\Delta t \cdot \Delta f} \int_{-\Delta t / 2}^{\Delta t / 2} \frac{S_{1}}{\Delta f}\left(t, f+\frac{\alpha}{2}\right) \frac{S_{1}^{*}}{\Delta f} \\
\times\left(t, f-\frac{\alpha}{2}\right) d t, \\
\frac{S_{1}}{\Delta f}(t, z)=\int_{-\Delta t / 2}^{\Delta t / 2} s(z) e^{-i 2 \lambda z t} d z,
\end{gathered}
$$

where $S_{1}$ represents the complex envelop of the band pass components of LU's signal $s(t)$ with centre frequency $z$ and 


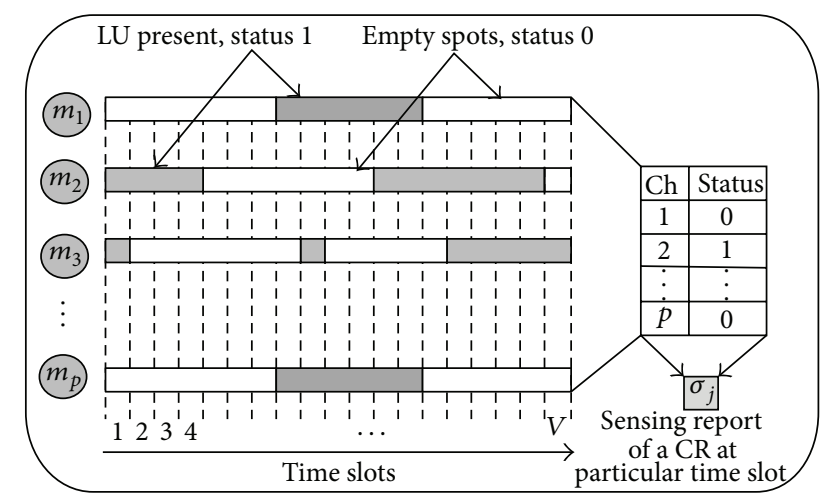

FIgURE 2: Random LUs activity: 0 represents idle state and 1 represents the busy state of a channel.

bandwidth $\Delta f$. The spectral components of the LU's signal can be measured in ideal condition using the following [33]:

$$
S_{p}^{\alpha}(f)=\lim _{\Delta f \rightarrow \infty \Delta t \rightarrow \infty} \lim _{s} S_{s}^{\alpha}(f)_{\Delta t} .
$$

The decision regarding cyclic frequency is based on the value of the coherence function given in the following [34]:

$$
D_{s}^{\alpha i}(f)=\frac{S_{s}^{\alpha i}(f)}{\left[S_{s}(f+(\alpha i / 2)) S_{s}(f-(\alpha i / 2))\right]^{1 / 2}} .
$$

The coherence function is compared with a threshold value. If it provides a higher value than the threshold, then the $\mathrm{LU}$ is present over the channel of frequency $f$ otherwise the $\mathrm{LU}$ is considered to be absent.

2.2.2. Mean Idle Time Prediction (MITP). It is very important for CRNs to select a control-channel with higher stability, that is, longer idle probability. The stable control-channel reduces the collisions with LUs and helps CRs to save a significant amount of retransmission power, which is consumed during reselection of the control-channel. Each reselection consumes power by rerunning of REPO algorithm. Hence, the initial selection should be made perfect under the given condition (availability of sensing information). The spectrum sensing is performed at a regular interval [35] and within each interval the $\mathrm{CR}$ forwards its observation to the respective CRBS. The sensing vector of a CR can be represented using

$$
\sigma_{j}=\left\{b_{1}, b_{2}, b_{3}, \ldots, b_{p}\right\},
$$

where $b_{k}$ represents the on-off state of the $k$ th channel at $j$ th time slot. Figure 2 explains the idle busy slots and the mechanism to acquire $\sigma_{j}$.

The base station of a particular intranetwork compares the current states of the channel with the states of previous time slot $(j-1)$. Only the different state values are transmitted to the CCM. This helps to reduce the control information between local base station and the CCM. The sensing report of each local CR base station can be represented using the expression given in

$$
\xi_{j}^{i}=\left\{c_{1}, c_{2}, c_{3}, \ldots, c_{x}\right\} \quad \text { for } b_{j} \neq b_{j-1}, x \leq p,
$$

where $\xi_{j}^{i}$ indicates the sensing reports from a local CR base station. Usually, the value of $x$ is less than $p$ until there is a rapid change in the arrival pattern of the CR. The CCM maintains the record of LUs activity over different channels for $v$ time slots. The history of the LU can be represented mathematically using

$$
\mathrm{H}_{p v}=\left[\begin{array}{cccc}
h_{11} & h_{12} & \cdots & h_{1 v} \\
h_{21} & h_{22} & \cdots & h_{2 v} \\
\vdots & \vdots & \vdots & \vdots \\
h_{p 1} & h_{p 2} & \cdots & h_{p v}
\end{array}\right],
$$

where $H$ represents the history matrix for holding the LU activity of $p$ channels over $v$ time slots and each element of the matrix is a binary digit 0 or 1 indicating the presence or absence of the LU during $j$ th time slot on $k$ th channel. After formation of the history of LU activity on different channels, the next prime task is the estimation of the idle time of the channels. For the sake of simplicity, we vertically divide the history into different regions where $\delta_{1}$ represents the most recent and $\delta_{s}$ is the oldest region as shown in Figure 3.

Suppose that $\lambda=\left\{\lambda_{1}, \lambda_{2}, \lambda_{3}, \ldots, \lambda_{s}\right\}$ represents the consecutive number of idle slots in a row of the history table and $w=\left\{w_{1}, w_{2}, w_{3}, \ldots, w_{s}\right\}$ indicates the weights assigned to different regions of the history. The mean idle time of the channels can be predicted using the expression given in

$$
\gamma_{k}=\sum_{l=1}^{s} w_{l} \lambda_{l}, \quad \forall l
$$

where $\gamma_{k}$ indicates the mean idle time factor of a channel and characterizes the stability of the channel.

2.2.3. Quality of Channels. Existing schemes such as [36] assume control-channels with the same capacity and the same communication range. However, the nature of the channel varies significantly across space and time which makes this assumption impractical. Therefore, we incorporate the quality variations of control-channels. We assume that each CR transmits with the same power $P_{t}$. If $I(t)=\left\{I_{1}, I_{2}, \ldots, I_{p}\right\}$ indicates the interference and $C=\left\{C_{1}, C_{2}, \ldots, C_{p}\right\}$ is the achievable data rate, then the capacity of $k$ th channel can be computed as follows:

$$
C_{k}(t)=B_{k} \log _{2}\left(1+\bar{K} \frac{S_{k}}{I_{k}}\right),
$$

where $B_{k}$ is the bandwidth of the channel and $\bar{K}$ is the limiting factor which varies with the BER and it can be represented as $\bar{K}=-1.5 / \log (5 \times$ BER $)$.

\section{Knapsack Problem Formulation and Algorithm}

The control-channel selection problem is formulated using the $0-1 \mathrm{KP}$. The channel capacity, mean idle time, and BER of the channels are modeled as the value of the knapsack and the weight of the channels are assumed to be constant. 


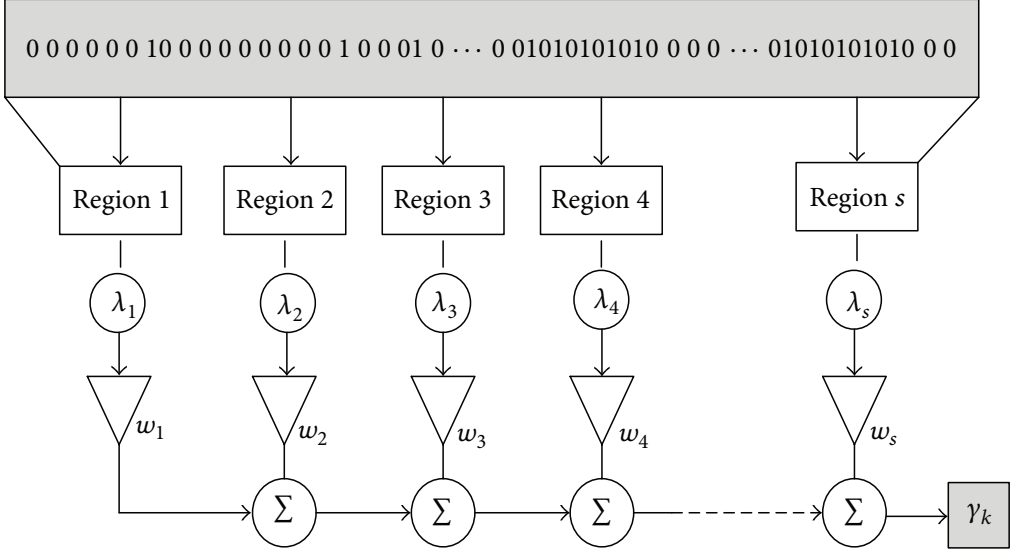

FIgURE 3: MITP framework.

Mathematically the knapsack based REPO can be represented as follows:

$$
\begin{aligned}
& \operatorname{Max} \quad \mathbf{A}=\sum_{k=1}^{p} Q_{k} x_{k} \\
& =\sum_{k=1}^{p}\left(\beta_{1} C_{k}+\beta_{2} \gamma_{k}+\beta_{3} \mu_{k}\right) x_{k}, \quad \forall k \\
& \text { subject to } \sum_{k=1}^{p} m_{k} x_{k} \leq \ell, \quad \forall k \text {, } \\
& C_{k} \cdot x_{k} \geq C_{\min }, \quad \forall k, \\
& \mathrm{BER}_{k} \cdot x_{k} \leq \mu_{k}\left(I_{k}\right), \quad \forall k, \\
& x_{k} \equiv\{0,1\}, \quad \forall k \text {, }
\end{aligned}
$$

where

$$
x_{k}=\left\{\begin{array}{ll}
1 & \text { selection } \\
0 & \text { otherwise }
\end{array} \quad \forall k\right. \text {. }
$$

Without loss of generality, we assume that

$$
\begin{gathered}
\max _{j}\left\{m_{k}\right\} \leq \ell, \quad \forall k, \\
\frac{Q_{1}}{m_{1}} \geq \frac{Q_{2}}{m_{2}} \geq \cdots \geq \frac{Q_{p}}{m_{p}}, \quad \forall k,
\end{gathered}
$$

where $Q$ represents the utility function or value factor, $\left\{\mu_{k}=\right.$ $1-\mathrm{BER}\}$, and $\beta_{1}, \beta_{2}$, and $\beta_{3}$ represent the weight or scaling factors for data capacity, idle time, and BER functions, respectively. The larger the value of the scaling factors, the stricter the priorities of the objective functions. The expression given in (13) illustrates the concept of backup channels in addition to the main control-channel (for current paper $\ell=3$, one main and two backup channels). The backup channels can play a crucial role in minimizing the alternate channel search time in the case of the sudden appearance of the LU over the selected control-channel. To further cater the random appearance of the LUs, we provide second alternate controlchannel. By having two backup channels, the CRs have better opportunities to manage the control tasks. Equation (14) assures that the selected channel satisfies the minimum capacity for the control traffic. By introducing the capacity constraint, we can reduce the possibility of channel handovers that may occur due to the poor signal to interference ratio. Similarly, (15) assures that the selected control-channel will have higher reliability through the selection of least BER channels. Hence, the REPO scheme offers better quality control-channels with a longer idle time and reliability.

3.1. Continuous Relaxation and Upper Bound. We can find the upper bound for 0-1 KP using the continuous relaxation mechanism which can be obtained by replacing (16) with the $0 \leq x_{k} \leq 1$ for all $k$. In [37], Dantzig has proved that the optimal solution $\left(x^{*}\right)$ of the given problem can be obtained by locating the critical item $\theta$ that satisfies the following condition

$$
\begin{gathered}
\sum_{k=1}^{\theta-1} m_{k} \leq \ell \leq \sum_{k=1}^{\theta} m_{k}, \\
\theta=\min \left\{k: \sum_{k 2=1}^{k} m_{k 2}>\ell\right\},
\end{gathered}
$$

where $\theta$ indicates the first item inserted into knapsack and it does not fit there and we have $1 \leq \theta \leq p$.

Theorem 1. The optimal solution $\left(x^{*}\right)$ of the proposed KP is as follows:

$$
\begin{gathered}
x^{*}=1 \quad \text { for } k=1,2, \ldots, \theta-1, \\
x^{*}=0 \quad \text { for } k=\theta+1, \theta+2, \ldots, p, \\
x_{\theta}^{*}=\frac{\ell^{*}}{m_{\theta}},
\end{gathered}
$$


where

$$
\ell^{*}=\ell-\sum_{k=1}^{\theta-1} m_{k}
$$

Proof. An optimal solution $\left(x^{*}\right)$ of KP must be maximal in the sense that $\sum_{k=1}^{p} m_{k} x_{k}=\ell$. Based on the assumption given in (18), now, let $x^{\circ}$ represent the optimal solution of the KP. In addition, suppose that $x_{k}^{\circ}<1$ for some $k<\theta$; then there must be $x_{y}^{\circ}>x_{y}^{*}$ for at least one item $y \geq \theta$. For a sufficiently small $\delta>0$, we could increase the value of $x_{k}^{\circ}$ by $\delta$, and $x_{y}^{\circ}$ can be decreased by $\delta m_{k} / m_{y}$. Therefore, augmenting the value of the objective function of $\delta\left(Q_{k}-Q_{y} m_{k} / m_{y}\right)>0$ as $\delta\left(Q_{k}-Q_{y} m_{k} / m_{y}\right)>0$ as $Q_{k} / m_{k}>Q_{y} / m_{y}$ which is clearly a contradiction to the original assumption. Similarly, we can prove that $x_{k}^{\circ}>0$ for $k>\theta$. Hence, $x_{\theta}^{*}=\ell^{*} / m_{\theta}$ using maximality. The optimal solution of the KP can be represented as follows [38]:

$$
\mathbf{A}\left(x^{*}\right)=\sum_{k=1}^{\theta-1} m_{k}+\left\lfloor\ell^{*} \frac{Q_{\theta}}{m_{\theta}}\right\rfloor .
$$

Hence, the valid upper bound of the proposed scheme based on the integrality of $Q_{k}$ and $x_{k}$ is

$$
U_{b}=\sum_{k=1}^{\theta-1} m_{k}+\left\lfloor\ell^{*} \frac{Q_{\theta}}{m_{\theta}}\right\rfloor .
$$

3.2. Dynamic Programming Solution for the Knapsack Problem. Dynamic programming (DP) is a powerful tool for solving the optimization problems such as knapsack problems [39]. In DP, there is a need to construct an array $Z[k, w]$, where $k$ and $w$ are integers satisfying $1 \leq k \leq p$ and $0 \leq$ $w \leq \ell$, respectively. An element of $Z[k, w]$ can be computed as follows:

$$
\begin{gathered}
Z[k, w]=\max \left(Z[k-1, w], Q_{k}+Z\left[k-1, w-\ell_{k}\right]\right) \\
\text { for } 1 \leq k \leq p, \quad 0 \leq w \leq \Omega \\
Z[0, w]=0 \quad \text { for } 0 \leq w \leq \Omega \\
Z[k, w]=-\infty \quad \text { for } w<0
\end{gathered}
$$

where $Q_{k}$ is the utility function associated with the $k$ th channel indicating the quality of the channel in terms and $\ell_{k}$ is the weight $k$ th channel and it is equal to 1 . Equation (24) holds only for those channels which can be selected based on their quality and stability. Each element of $Z$ stores the accumulated sum of the utility function for the selected channel, where the total number of channels (main CC and backup CC) is equal to $\ell$.

3.2.1. Optimal Substructure Property. Let $\left(x_{1}, x_{2}, x_{3}, \ldots, x_{k}\right)$ represent the optimal solution of the proposed KP of REPO scheme depicted in Section 3. And let $\left(x_{2}, x_{3}, \ldots, x_{k}\right)$ indicate the optimal solution for the subproblem of the proposed system which can be represented mathematically using (27) [36].

Consider the following:

$$
\begin{array}{ll}
\operatorname{Max} & \mathbf{A}^{\prime}=\sum_{k 1=2}^{p} Q_{k 1} x_{k 1}, \quad \forall k 1 \\
\text { subject to } & \sum_{k 1=2}^{p} m_{k 1} x_{k 1} \leq \ell, \quad \forall k 1, \\
& C_{k 1} \cdot x_{k 1} \geq C_{\min }, \quad \forall k 1, \\
& \mathrm{BER}_{k 1} \cdot x_{k 1} \leq \mu_{k 1}\left(I_{k 1}\right), \quad \forall k 1, \\
& x_{k 1} \equiv\{0,1\}, \quad \forall k 1 .
\end{array}
$$

Now, we also assume that $\left(u_{2}, u_{3}, \ldots, u_{k}\right)$ is also an optimal solution to the subproblem (19)-(23) rather than $\left(x_{2}, x_{3}, \ldots, x_{k}\right)$. Thus we show that

$$
\begin{aligned}
& \sum_{k 1=2}^{p} Q_{k 1} x_{k 1}<\sum_{k 1=2}^{p} Q_{k 1} u_{k 1}, \quad \forall k 1, \\
& m_{1} x_{1}+\sum_{k 1=2}^{p} m_{k 1} u_{k 1} \leq \ell, \quad \forall k 1 .
\end{aligned}
$$

Hence the result comes

$$
\begin{gathered}
Q_{1} x_{1}+\sum_{k 1=2}^{p} Q_{k 1} u_{k 1}>\sum_{k 1=2}^{p} Q_{k 1} x_{k 1}, \quad \forall k 1, \\
m_{1} x_{1}+\sum_{k 1=2}^{p} m_{k 1} u_{k 1} \leq \ell, \quad \forall k 1 \\
C_{k 1} \cdot u_{k 1} \geq C_{\min }, \quad \forall k 1, \\
\operatorname{BER}_{k 1} \cdot u_{k 1} \leq \mu_{k 1}\left(I_{k 1}\right), \quad \forall k 1 \\
x_{u 1} \equiv\{0,1\}, \quad \forall k 1 .
\end{gathered}
$$

The given expression suggests that $\left(u_{2}, u_{3}, \ldots, u_{k}\right)$ is an optimal solution which proves that the $0-1 \mathrm{KP}$ has substructure property which formulates the recursive formula given in (24).

3.2.2. Space and Time Complexity. The recursive function described in (17) requires the integer weights $m_{k}$ of the selected channels. Based on this condition, the time and space complexity of the proposed scheme is $O(k \ell)$. By adopting the robust programming mechanism, the space complexity can be reduced to $O(\ell)$. For larger values of $\ell$, the time complexity of the proposed scheme increases and the time complexity of the worst-case scenario is $O\left(k 2^{k}\right)$ when $\ell=2^{k}$. However, in [40], the authors propose a combo algorithm to improve the performance of the KP algorithm. They combine the DP with tight bounds to avoid the worst-case scenario and allow the system to reach an optimal solution within 


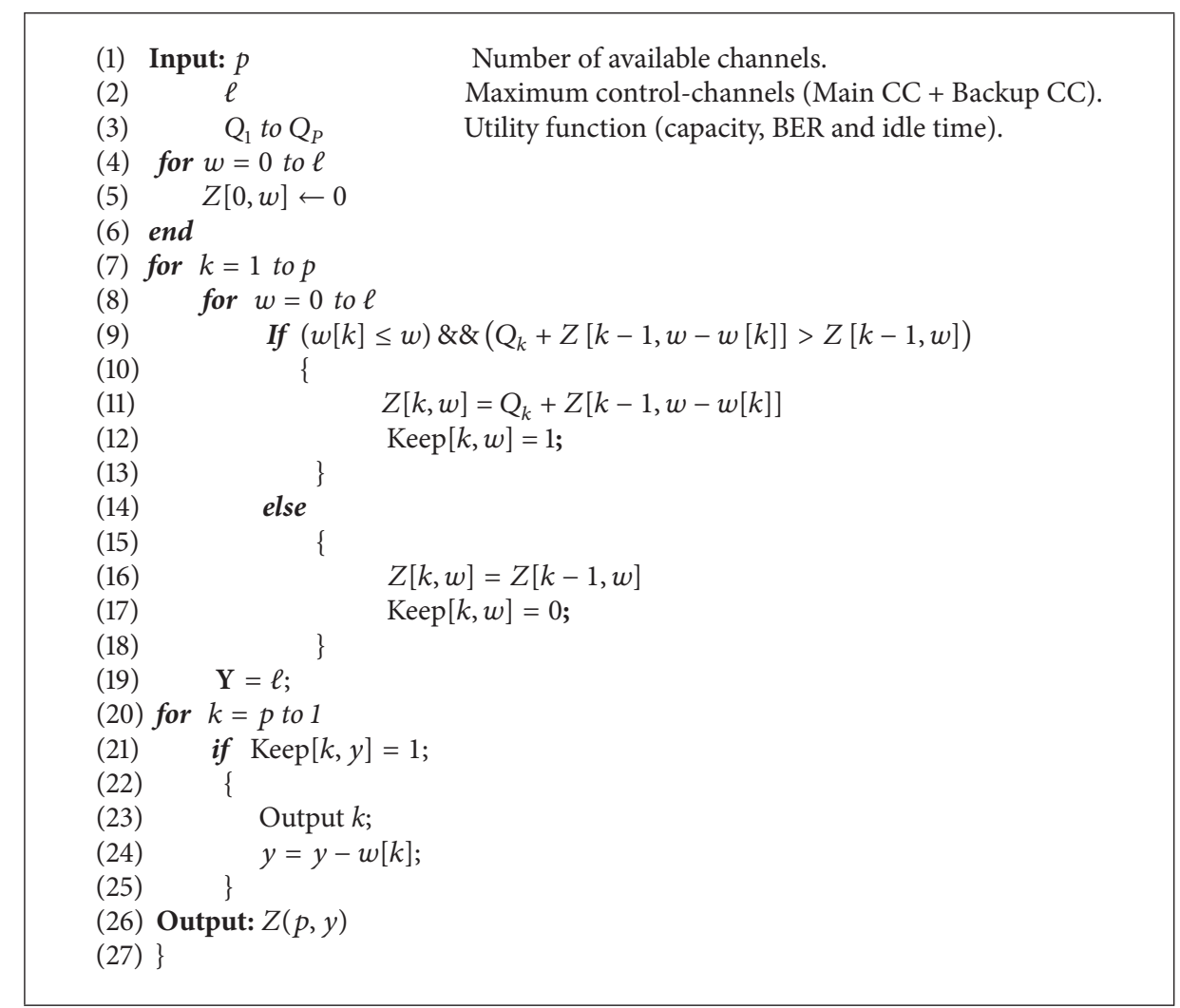

Algorithm 1: Dynamic programming code for reliable, efficient, and power optimized (REPO) scheme.

an acceptable time. Algorithm 1 explains the steps involved in the programming of the proposed REPO scheme. A keep array is introduced to monitor the quality of the selected channels. Hence, the optimal channels are selected using the DP approach.

3.3. Hand-Off Delay. To find the mathematical relationship for estimating the total hand-off delay incurred, suppose that $\Delta_{\text {tot }}$ is the delay incurred during an application round with certain LU activity to manage the control information for the data transmission of the CRs at a particular time. Let $\Delta_{w i}$ denote the time span for the smooth operation over the selected control-channel without interruptions from LUs and $\phi$ is the total occurrences of the LUs for a given application round. The total time transmission delay for the transmission of control information can be computed using

$$
\begin{gathered}
\Delta_{\text {tot }}=\Delta_{w i}+\phi_{1} \cdot \Delta_{b 1}+\phi_{2} \cdot \Delta_{b 2}+\Delta_{r f}, \\
\phi=\phi_{1}+\phi_{2},
\end{gathered}
$$

where $\phi_{1}$ and $\phi_{2}$ represent the occurrence of the LUs arrival on the main and first backup control-channel, respectively. The term $\Delta_{r f}$ in (30) indicates the time consumed during the alternate channel search (reselection of the control-channels). It is equal to the time consumed during the channel sensing and channel selection time. Contrary to the second and third factors, $\Delta_{r f}$ causes more delay, where $\Delta_{r f} \gg \phi_{s} \cdot \Delta_{b s}$.
TABLE 1: Simulation parameters.

\begin{tabular}{lc}
\hline Parameters & Value \\
\hline Transmission power $\left(P_{t}\right)$ & $30 \mathrm{dBm}$ \\
Noise variance $\left(\sigma^{2}\right)$ & $0.2 \sim 0.7$ \\
History slots $(v)$ & 100 \\
History samples per region & {$[20203030]$} \\
Channels $(p)$ & 100 \\
LU activity & $0 \sim 50 \%$ \\
{$\left[\beta_{1}, \beta_{2}, \beta_{3}\right]$} & {$[0.2,0.5,0.3]$} \\
\hline
\end{tabular}

\section{Simulation Results}

In this section, we explain the steps used to carry out the experimental results and evaluate the performance of REPO scheme through different comparisons. MATLAB 7.0 is used for simulation results. Although, we have shown the simulation results for specific parameters values, the proposed scheme can perform equally well for more generic cases. To improve the clarity and make the reading easier, we have shown the comparisons across four important parameters: (1) average collision rate, (2) average hand-off delay, (3) average power consumption, and (4) reliability. Table 1 presents the different simulation parameters that are used to acquire results. 
TABLE 2: Mean idle time of selected channels.

\begin{tabular}{lcccc}
\hline \multirow{2}{*}{ Channel type } & \multicolumn{4}{c}{ Mean idle time of channels $\left(\gamma_{k}\right)$} \\
& $p=25$ & $p=50$ & $p=75$ & $p=100$ \\
\hline Main CC & 7.76 & 9.03 & 9.65 & 10.60 \\
Backup 1 CC & 6.38 & 7.43 & 8.01 & 8.92 \\
Backup 2 CC & 5.64 & 6.57 & 7.03 & 7.51 \\
\hline
\end{tabular}

Table 2 presents the outcome of REPO scheme based on predicted idle time of the selected control-channels for four different numbers of available channels. The Monte Carlo Principle is used to simulate the result for 100 iterations and then the mean idle times of the main and two backup channels are presented. The main control-channel can provide up to 10 idle slots for the operation of the CRs with the availability of $P \geq 75$.

4.1. Average Collisions with LUs. In this section, we show the performance gain of our proposed REPO scheme over random channel selection (RCS) and greedy channel selection (GCS) schemes based on the average collision rate with the LUs. We have shown the results by varying different parameters of the simulation and try to highlight the advantages of the proposed scheme. The average values of the LU's collision rate are computed after performing 100 iterations and then the average values are shown for comparison.

Figure 4(a) shows a comparison based on the collision rate across different application rounds. An application round represents the validity of the current selection in terms of the number of time slots. Each application round is five slots ahead from the previous application round. There are two key observations in the result. Firstly, for the first two application rounds, the REPO scheme shows [37.2\%, 29.3\%] less number of collisions as compared to the GCS scheme and the performance of the REPO is 3 times better in comparison with RCS scheme. Secondly, it provides information regarding the optimal application rounds for the operation of the proposed scheme (application rounds $\leq 3$ ). On the third application round, REPO offers 26.3 and $65.12 \%$ less number of collisions with LUs as compared to the GCS and RCS schemes.

Figure 4(b) compares the proposed and existing schemes for different numbers of available channels. The REPO scheme offers 2.58 times less number of collisions with the increase of the available channels from 10 to 100. For example, when control-channel is selected from the pool of 50 channels by DCS and RCS schemes, they offer on average $21.39 \%$ to $61.17 \%$ greater number of collisions as compared to the REPO scheme. This is due to the fact that when CRNs have more channels in the poll (sensing results), the probability to find good channels (in terms of capacity, error rate, and idle time) increases.

Figure 4(c) illustrates the comparison of the REPO scheme with the existing schemes across different percentages of LU activity for three different application rounds (application round $1=5$ free slots, application round $2=$ 10 free slots, and application round $3=15$ free slots). The REPO shows better performance for all three application rounds. For example, during application round 3, the REPO shows [ $16.8 \% 10.89 \%],\left[\begin{array}{lll}35.48 \% & 43.58 \%\end{array}\right]$ lower collision rate for $20 \%$ and $50 \%$ LU activity over different channels, respectively. The performance gain of the REPO scheme is also very significant for other percentages of LU activity. Similarly, Figure 4(d) also validates the performance gain of the proposed REPO over existing schemes across different LU activities. We plot the result for three different cases $(p=25$, $p=50$, and $p=75$ ). Again, the REPO scheme shows better performance than the GCS and RCS schemes for all cases. Hence, the REPO is an effective scheme to cater the random LU activity.

4.2. Average Hand-Off Delay. In this section, we compare our scheme with the RCS and GCS schemes considering the average channel hand-off time. The performance gain achieved through the use of the backup channels is also presented. The average values of the hand-off delay are plotted after performing 100 iterations. Moreover, we also show a comparison with the two popular hand-off strategies: (1) always stay (AS) and (2) always change (AC). In the AS scheme, the CR stays on the same channel and waits for LU to finish its transmission until the channel becomes available again. However, in the AC scheme, the CR always changes the channel for its remaining transmission.

Figure 5(a) highlights the advantages of the proposed scheme with the use of the backup channels. The performance of the REPO is equally better for all application rounds. However, the backup channels improve the performance of REPO in an exponential pattern. For example, during application round 3, the REPO shows $26 \%$ and $65 \%$ lower hand-off delay compared to the GCS and RCS scheme. By using a single backup channel, the delay is reduced 3 times in comparison with GCS and 4 times compared to the RCS scheme. The gain factor is more significant with the use of a second backup control-channel.

Figure 5(b) illustrates the advantages of the backup channels across different LU activity. The REPO scheme significantly reduces the hand-off delay as compared to the RCS and GCS scheme. For example, consider the $30 \%$ LU activity and the REPO scheme with 2 backup channels; the GCS and RCS schemes offer 4.2 times and 5 times higher hand-off delay as compared to the REPO scheme. Hence, the REPO scheme performs equally better than the GCS and RCS schemes in reducing the hand-off delay time. To further extend the comparison with the existing schemes, we evaluate the REPO scheme with AS and AC scheme as shown in Figure 5(c). The REPO scheme again performs significantly better than these two schemes. For example, when the LU activity is 30\%, the REPO scheme shows 4.6 times and 5.4 times less delays compared to the AC and AS hand-off schemes. Hence, the proposed REPO scheme can perform better than many of the existing channel selection schemes.

4.3. Average Power Consumption. Figure 6 illustrates the performance gain of the REPO scheme over GCS and RCS schemes in terms of power consumption. We simulate the result for two different application rounds and show the bar graph for the 1st and 2nd application round (cases 


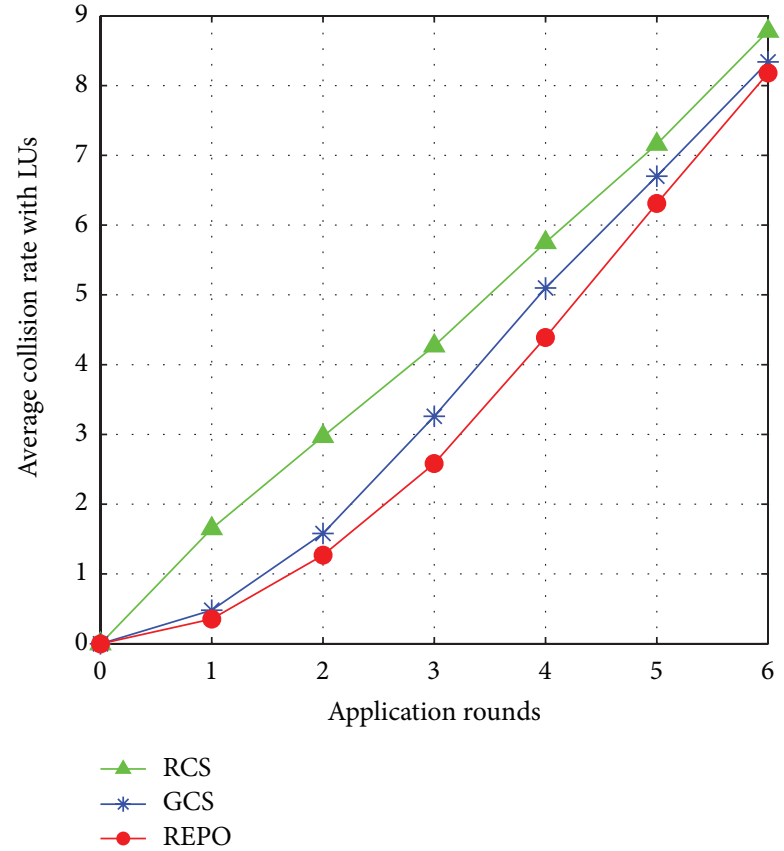

(a)

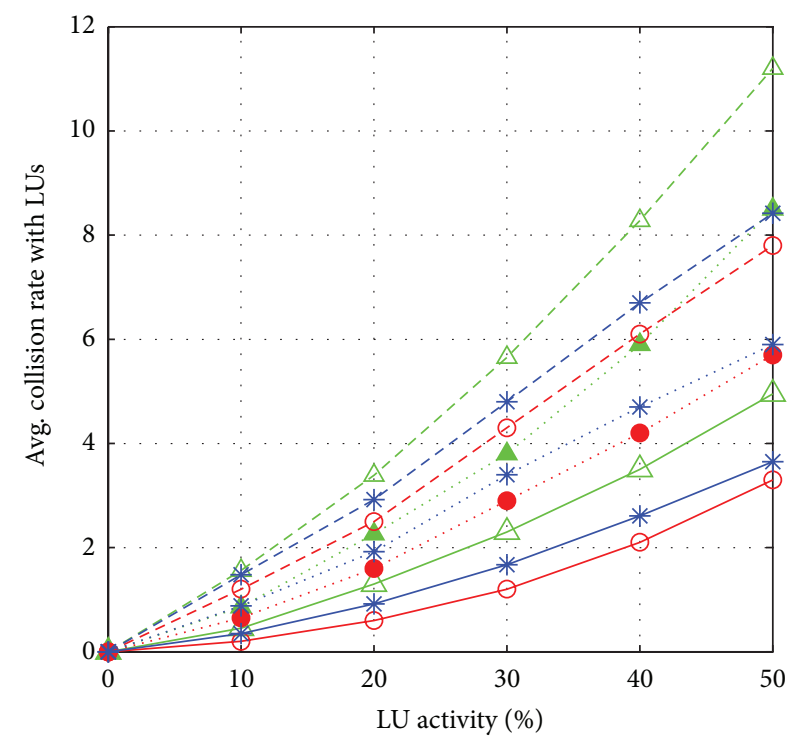

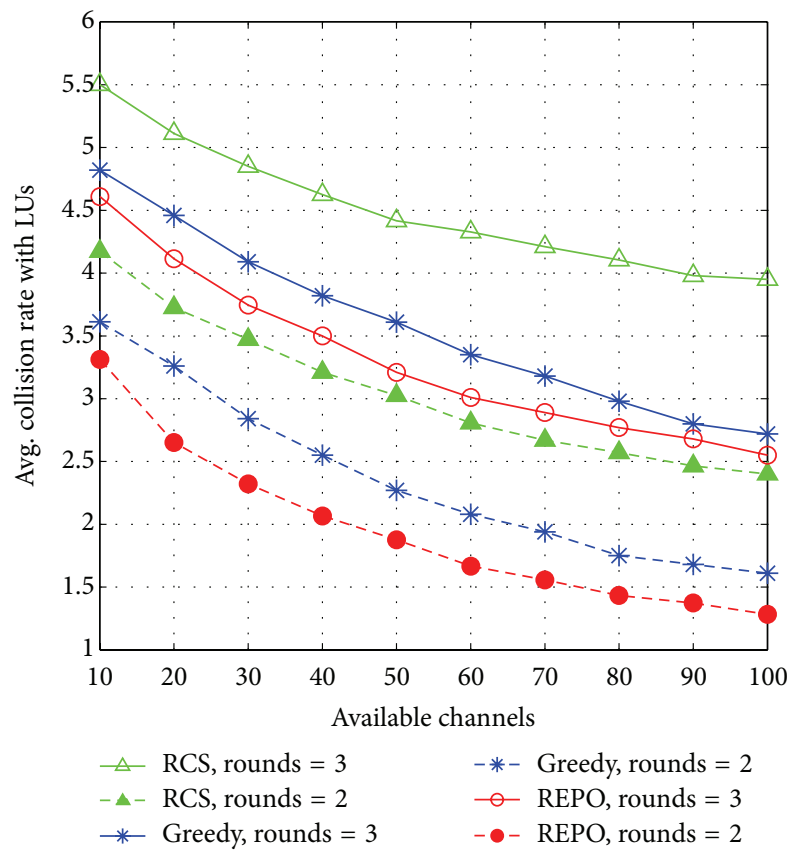

(b)
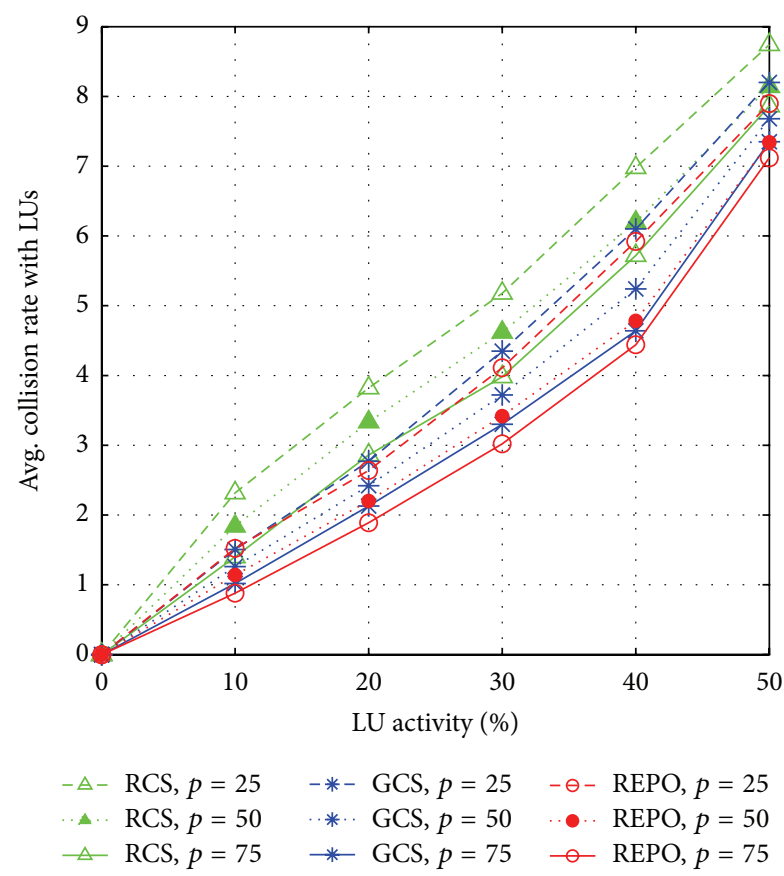

(d)

FIGURE 4: Comparison based on the collision with LUs (a) for different application rounds, (b) for different available channels, (c) for different percentage of LU activity, and (d) for different LU activity with three different cases of available channels. 


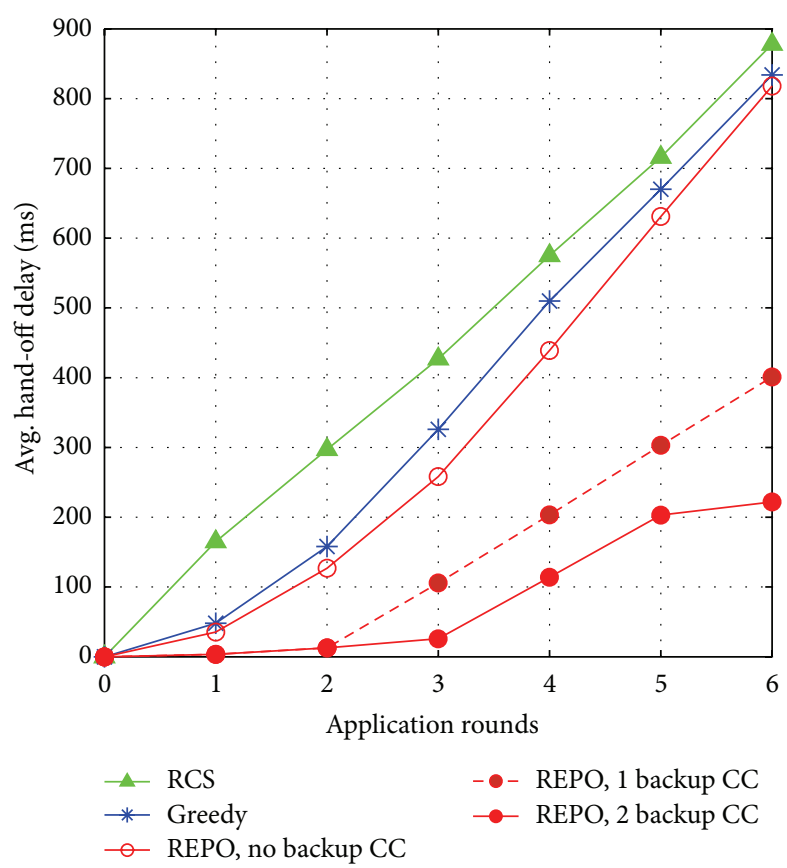

(a)

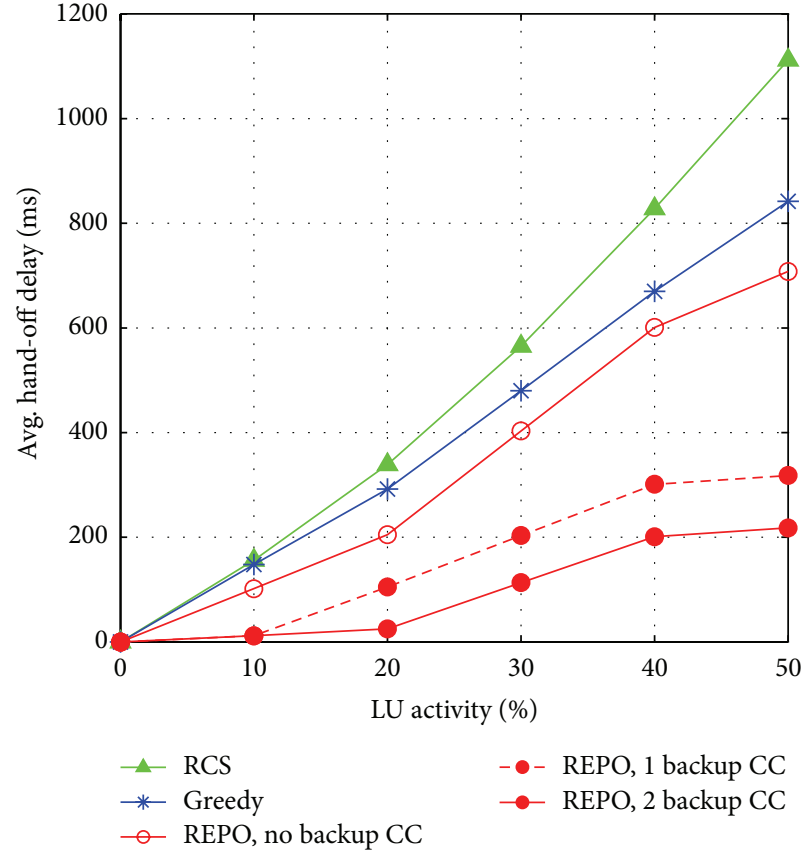

(b)

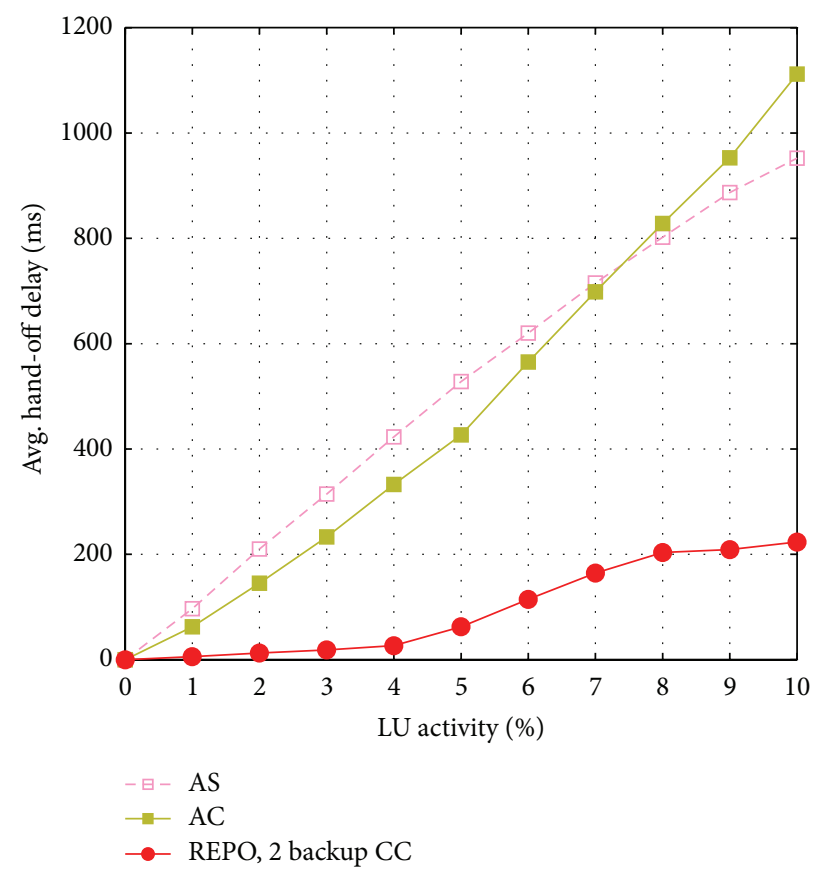

(c)

FIGURE 5: Hand-off delay based comparison, (a) for different application rounds, (b) for different LU activity, and (c) for AS and AC schemes.

in Figure 5(c)) assuming that $100 \mathrm{mw}$ is consumed for the retransmission of control data in case of collision with the LUs. The REPO scheme performs equally well to save transmission power. For example, consider 30\% LU activity and application round 2 where the REPO saves $13.79 \%$ and $34.03 \%$ power as compared to the GCS and RCS scheme. Therefore, the DCS scheme is also useful for power sensitive application of the CRN such as cognitive radio sensor networks (CRSNs).
4.4. Reliability. This section shows performance gain based on the reliability factor $\mu(1-\mathrm{BER})$. The BER of channels are varied from $10^{-1}$ to $10^{-16}$ and the normalized average reliability values of the control-channels selected using existing schemes and REPO scheme are plotted in Figure 7. The weight given to the reliability factor in the utility function of (12) assures the selection of highly reliable channels with minimal BER. The comparison is shown for six different 


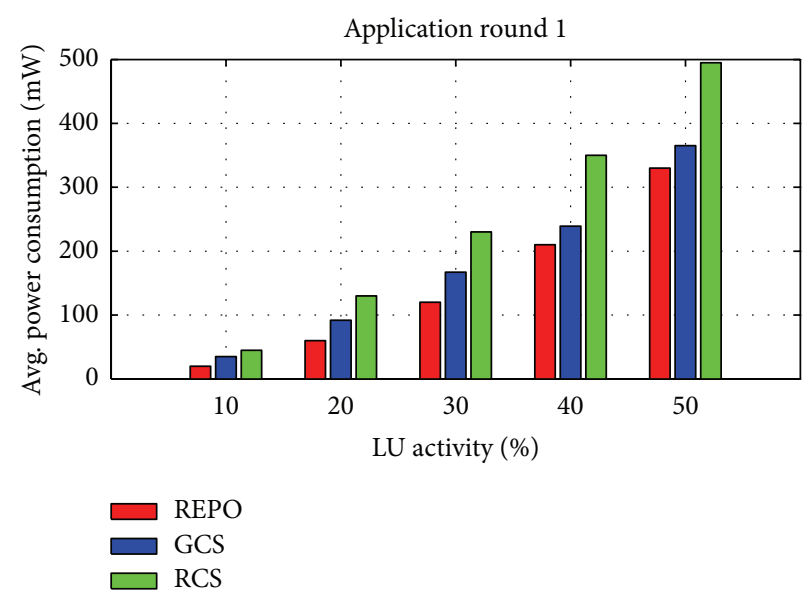

(a)

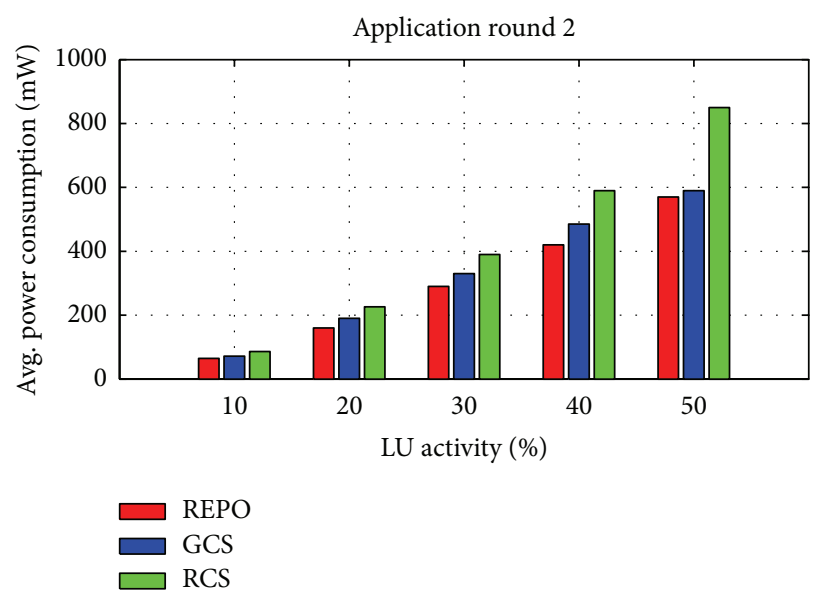

(b)

FIGURE 6: Comparison based on the power consumption.

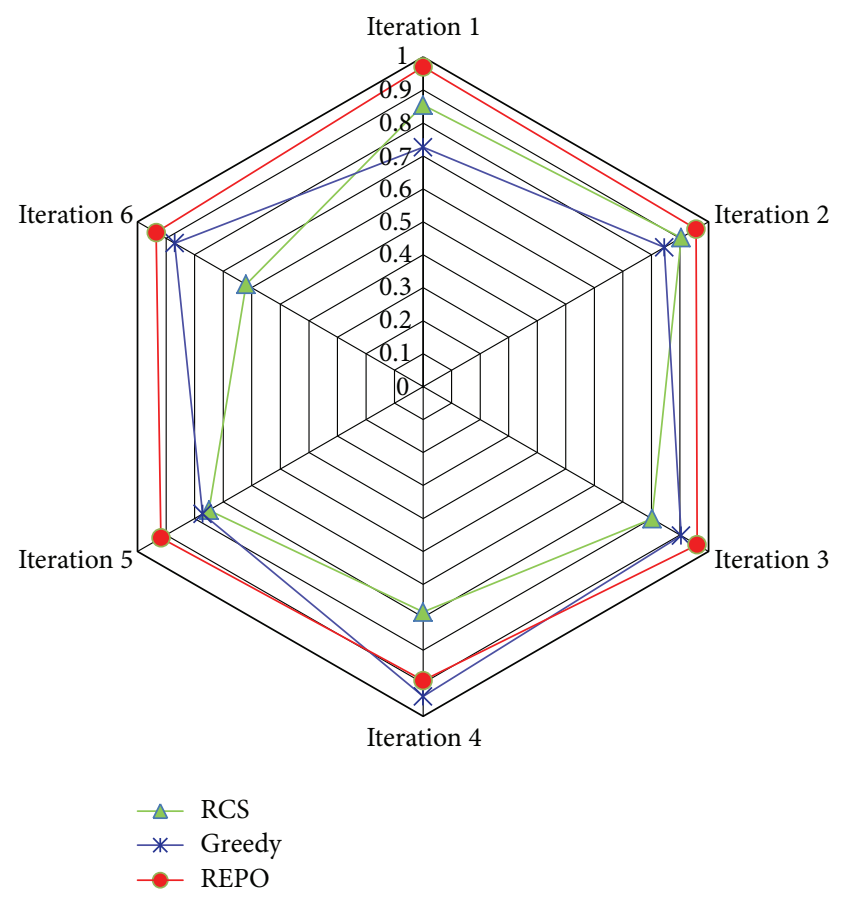

FIgURE 7: Comparison based reliability.

iterations. In each iteration, the control-channel is selected 100 times and then the average values of the reliability function are shown in the graph. The value 1 represents the $\mathrm{BER}=10^{-16}$ and value 0 corresponds to the BER level $10^{-1}$. We divide the boundary between 0 and 1 in 16 levels where each level is 0.0625 ahead from its previous level, that is, $10^{-15}$ corresponds to 0.9375 . The REPO scheme selects the channels with the reliability range of [0.89 to 0.97], whereas the GCS and RCS schemes select the channels with the reliability range of [0.726 to 0.94$]$ and [0.62 to 0.90$]$, respectively. Hence, the proposed REPO scheme selects channels with the least BER values and offer a better environment for CRs.

\section{Conclusion}

The control-channel selection scheme plays a key role in the effective operation of CRNs. However, the variations in the spectrum across capacity and occupancy are the crucial challenges in the design of the control-channel selection scheme. In this paper, we propose a REPO scheme for the effective and optimal management of the control traffic in CRNs. We formulate the control-channel selection problem using the KP and employ the dynamic programming approach to select the most stable and better quality control-channels for the smooth operation of CRs. We incorporate the mean idle time (to cater for the random appearance of the LU), $\mathrm{BER}$, and channel capacity (to counter the variation at the physical layer) as the selection criteria by introducing these three factors inside the KP. To reduce the alternate channel search time, we introduce the concept of backup controlchannels and employ two backups to reduce the alternate channel search time.

The simulation results show that the proposed REPO scheme provides stable control-channels to the CRs under the desired constraints of capacity and error rate. The REPO scheme reduces collisions with the LUs and minimizes the channel hand-off delay. Moreover, it reduces significant amount of retransmission power when compared to the RCS and GCS schemes. The reliability analysis shows that the REPO scheme selects the channel with the least BER values and offers a better environment under noisy conditions.

One of the limitations of the proposed scheme is the initial selection of the control-channel in the ISM band. Although the proposed system quickly moves on to the dynamic control selection, it relies, to a small extent, on the fixed channel selection. An alternative dynamic controlchannel for network initialization can be a good useful subject 
for future work. Secondly, the computation of the mean idle time requires history of the PUs and storage reduction for the PU history samples seems to be an interesting topic of study. Moreover, a similar concept can be applied to ad hoc networks and time-critical cognitive radio sensor networks (CRSNs).

\section{Conflict of Interests}

The authors declare that there is no conflict of interests regarding the publication of this paper.

\section{Acknowledgment}

This research was supported by the MKE (The Ministry of Knowledge Economy), Korea, under the Convergence-ITRC (Convergence Information Technology Research Center) support program (NIPA-2013-H0401-13-1003) supervised by the NIPA (National IT Industry Promotion Agency) and the Basic Science Research Program through the National Research Foundation of Korea (NRF) funded by the Ministry of Education, Science and Technology (2012009449).

\section{References}

[1] B. F. Lo, I. F. Akyildiz, and A. M. Al-Dhelaan, "Efficient recovery control channel design in cognitive radio ad hoc networks," IEEE Transactions on Vehicular Technology, vol. 59, no. 9, pp. 4513-4526, 2010.

[2] S. Aslam and K. G. Lee, "Fair, efficient, and power-optimized spectrum sharing scheme for cognitive radio networks," EURASIP Journal on Wireless Communication Networking, vol. 2011, Article ID 201, 2011.

[3] S. Aslam and K. G. Lee, "CSPA: channel selection and parameter adaptation scheme based on genetic algorithm for cognitive radio Ad Hoc networks," EURASIP Journal on Wireless Communication Networking, vol. 2012, Article ID 349, 2012.

[4] B. Stundag and O. Orcay, "A pattern construction ccheme for neural network-based cognitive communication," Entropy, vol. 13, no. 1, pp. 64-81, 2011.

[5] I. F. Akyildiz, W. Y. Lee, M. C. Vuran, and S. Mohanty, "NeXt generation/dynamic spectrum access/cognitive radio wireless networks: a survey," Computer Networks, vol. 50, no. 13, pp. 2127-2159, 2006.

[6] R. Wang, G. P. Liu, W. Wang, D. Rees, and Y. B. Zhao, " $\mathrm{H}_{\infty}$ control for networked predictive control systems based on the switched Lyapunov function method," IEEE Transactions on Industrial Electronics, vol. 57, no. 10, pp. 3565-3571, 2010.

[7] R. Wang, G. P. Liu, W. Wang, D. Rees, and Y. B. Zhao, "Guaranteed cost control for networked control systems based on an improved predictive control method," IEEE Transactions on Control Systems Technology, vol. 18, no. 5, pp. 1226-1232, 2010.

[8] X. M. Sun, G. P. Liu, W. Wang, and D. Rees, "Stability analysis for networked control systems based on event-time-driven mode," International Journal of Control, vol. 82, no. 12, pp. 2260-2266, 2009.

[9] X. M. Sun, G. P. Liu, W. Wang, and D. Rees, "Stability analysis for networked control systems based on average dwell time method," International Journal of Robust and Nonlinear Control, vol. 20, no. 15, pp. 1774-1784, 2010.
[10] Q. Su, X. M. Sun, and J. Zhao, "Switching tracking control for planar systems with transient performance constraints," Acta Automatica Sinica, vol. 39, no. 6, pp. 919-925, 2013.

[11] B. F. Lo, "A survey of common control channel design in cognitive radio networks," Physical Communication, vol. 4, no. 1, pp. 26-39, 2011.

[12] H. A. B. Salameh, M. M. Krunz, and O. Younis, "MAC protocol for opportunistic cognitive radio networks with soft guarantees," IEEE Transactions on Mobile Computing, vol. 8, no. 10, pp. 1339-1352, 2009.

[13] D. Cabric, S. Mishra, D. Willkomm, R. Brodersen, and A. Wolisz, "A cognitive radio approach for usage of virtual unlicensed spectrum," in Proceedings of the 14th IST Mobile and Wireless Communications Summit, Dresden, Germany, 2005.

[14] A. Masri, C.-F. Chiasserini, and A. Perotti, "Control information exchange through UWB in cognitive radio networks," in Proceedings of the IEEE 5th International Symposium on Wireless Pervasive Computing (ISWPC '10), pp. 110-115, Modena, Italy, May 2010.

[15] M. E. Sahin and H. Arslan, "System design for cognitive radio conmunications," in Proceedings of the 1st International Conference on Cognitive Radio Oriented Wireless Networks and Communications (CROWNCOM '06), pp. 1-5, Mykonos Island, Greece, 2006.

[16] A. Masri, C. F. Chiasserini, C. Casetti, and A. Perotti, "Common control-channel allocation in cognitive radio networks through UWB communications," Journal of Computer and Networks, vol. 11, no. 6, pp. 710-718, 2012.

[17] D. Cabric, S. M. Mishra, and R. W. Brodersen, "Implementation issues in spectrum sensing for cognitive radios," in Proceedings of the Conference Record of the 38th Asilomar Conference on Signals, Systems and Computers, pp. 772-776, November 2004.

[18] D. Raychaudhuri and X. Jing, "A spectrum etiquette protocol for efficient coordination of radio devices in unlicensed bands," in Proceedings of the 14th IEEE 2003 International Symposium on Personal, Indoor and Mobile Radio Communications, (PIMRC '03), pp. 172-176, Beijing, China, September 2003.

[19] X. Jing and D. Raychaudhuri, "Proceedings of the Spectrum Co-existence of IEEE 802.11b and 802.16a networks using the CSCC etiquette protocol," in Proceedings of the 2005 1st IEEE International Symposium on New Frontiers in Dynamic Spectrum Access Networks, (DySPAN '05), pp. 243-250, Baltimore, Md, USA, November 2005.

[20] C. Han, J. Wang, Y. Yang, and S. Li, "Addressing the control channel design problem: OFDM-based transform domain communication system in cognitive radio," Computer Networks, vol. 52, no. 4, pp. 795-815, 2008.

[21] A. Ghasemi and E. S. Sousa, "Collaborative spectrum sensing for opportunistic access in fading environments," in Proceedings of the 2005 1st IEEE International Symposium on New Frontiers in Dynamic Spectrum Access Networks, (DySPAN '05), pp. 131136, Baltimore, Md, USA, November 2005.

[22] S. M. Mishra, A. Sahai, and R. W. Brodersen, "Cooperative sensing among cognitive radios," in Proceedings of the 2006 IEEE International Conference on Communications, (ICC '06), pp. 1658-1663, Istanbul, Turkey, July 2006.

[23] E. Axell, G. Leus, E. G. Larsson, and H. V. Poor, "Spectrum sensing for cognitive radio : state-of-the-art and recent advances," IEEE Signal Processing Magazine, vol. 29, no. 3, pp. 101-116, 2012.

[24] P. Bahl, R. Chandra, T. Moscibroda, R. Murty, and M. Welsh, "White space networking with Wi-Fi like connectivity," in 
Proceedings of the ACM Conference on Data Communication (SIGCOMM '09), pp. 27-38, Barcelona, Spain, August 2009.

[25] L. Lazos, S. Liu, and M. Krunz, "Mitigating control-channel jamming attacks in multi-channel ad hoc networks," in Proceedings of the 2nd ACM Conference on Wireless Network Security (WiSec '09), pp. 169-180, Zurich, Switzerland, March 2009.

[26] J. Zhao, H. Zheng, and G. H. Yang, "Spectrum sharing through distributed coordination in dynamic spectrum access networks," Wireless Communications and Mobile Computing, vol. 7, no. 9, pp. 1061-1075, 2007.

[27] T. Chen, H. Zhang, G. M. Maggio, and I. Chlamtac, "CogMesh: a cluster-based cognitive radio network," in Proceedings of the 2nd IEEE International Symposium on New Frontiers in Dynamic Spectrum Access Networks, pp. 168-178, Dublin, UK, April 2007.

[28] P. Bahl, R. Chandra, T. Moscibroda, R. Murty, and M. Welsh, "White space networking with Wi-Fi like connectivity," in Proceedings of the ACM Conference on Data Communication (SIGCOMM '09), pp. 27-38, Barcelona, Spain, August 2009.

[29] Y. R. Kondareddy and P. Agrawal, "Synchronized MAC protocol for multi-hop cognitive radio networks," in Proceddings of the IEEE International Conference on Communications (ICC '08), pp. 3198-3202, Beijing, China, May 2008.

[30] L. A. DaSilva and I. Guerreiro, "Sequence-based rendezvous for dynamic spectrum access," in Proceedings of the 3rd IEEE Symposium on New Frontiers in Dynamic Spectrum Access Networks (DySPAN '08), pp. 1-7, Chicago, Ill, USA, October 2008.

[31] P. D. Sutton, K. E. Nolan, and L. E. Doyle, "Cyclostationary signatures in practical cognitive radio applications," IEEE Journal on Selected Areas in Communications, vol. 26, no. 1, pp. 13-24, 2008.

[32] W. A. Gardner, "Signal interception: a unifying theoretical framework for feature detection," IEEE Transactions on Communications, vol. 36, no. 8, pp. 897-906, 1988.

[33] W. A. Gardner, "Spectral correlation of modulated signals: part I-analog modulation," IEEE Transactions on Communications, vol. 35, no. 6, pp. 584-594, 1987.

[34] W. A. Gardner, W. A. Brown, and C.-K. Chen, "Spectral correlation of modulated signals: part II-digital modulation," IEEE Transactions on Communications, vol. 35, no. 6, pp. 595601, 1987.

[35] C. Passiatore and P. Camarda, "A centralized inter-network resource sharing (CIRS) scheme in IEEE 802.22 cognitive networks," in Proceedings of the 10th IFIP Annual Mediterranean Ad Hoc Networking Workshop (Med-Hoc-Net '11), pp. 17-24, Sicily, Italy, June 2011.

[36] L. Lazos, S. Liu, and M. Krunz, "Spectrum opportunity-based control channel assignment in cognitive radio networks," in Proceedings of the 6th Annual IEEE Communications Society Conference on Sensor, Mesh and Ad Hoc Communications and Networks, (SECON '09), Rome, Italy, June 2009.

[37] G. B. Dantzig, "Discrete-variable extremum problems," Operations Research, vol. 5, pp. 266-277, 1957.

[38] T. H. Cormen, C. E. Leiserson, R. L. Rivest, and C. Stein, Introduction to Algorithms, MIT Press, Cambridge, Mass, USA, 2nd edition, 2001.

[39] X. Meng, Y. A. Zhu, and X. Wu, "Improved dynamic programming algorithms for the 0-1 knapsack problem," in Proceedings of the 3rd IEEE International Conference on Computer Science and Information Technology (ICCSIT '10), pp. 19-22, Chengdu, China, July 2010.
[40] S. Martello, D. Pisinger, and P. Toth, "Dynamic programming and tight bounds for the 0-1 knapsack problem," Research Report OR197/1, DEIS, University of Bologna, 1997. 


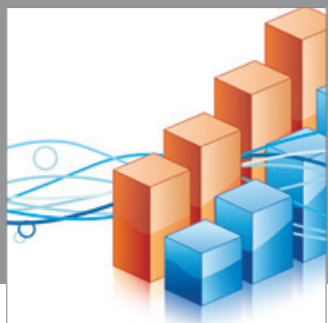

Advances in

Operations Research

mansans

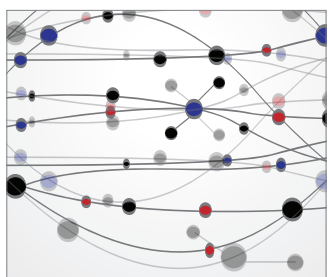

The Scientific World Journal
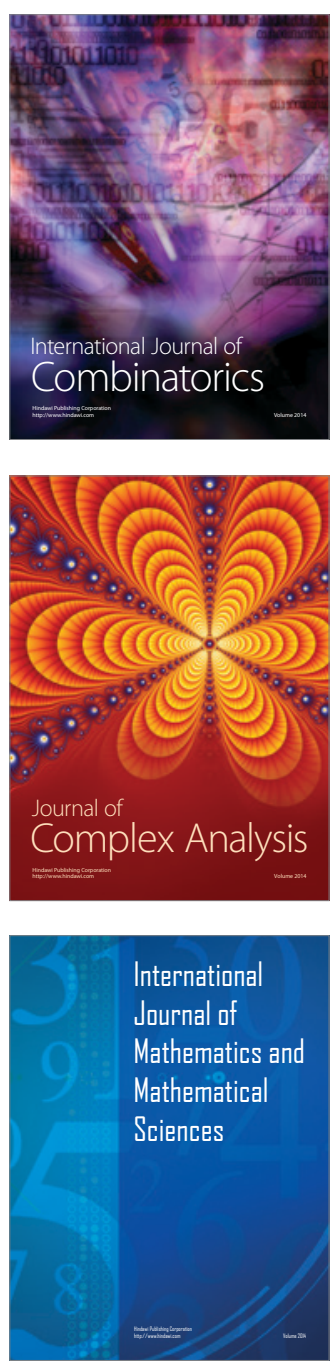
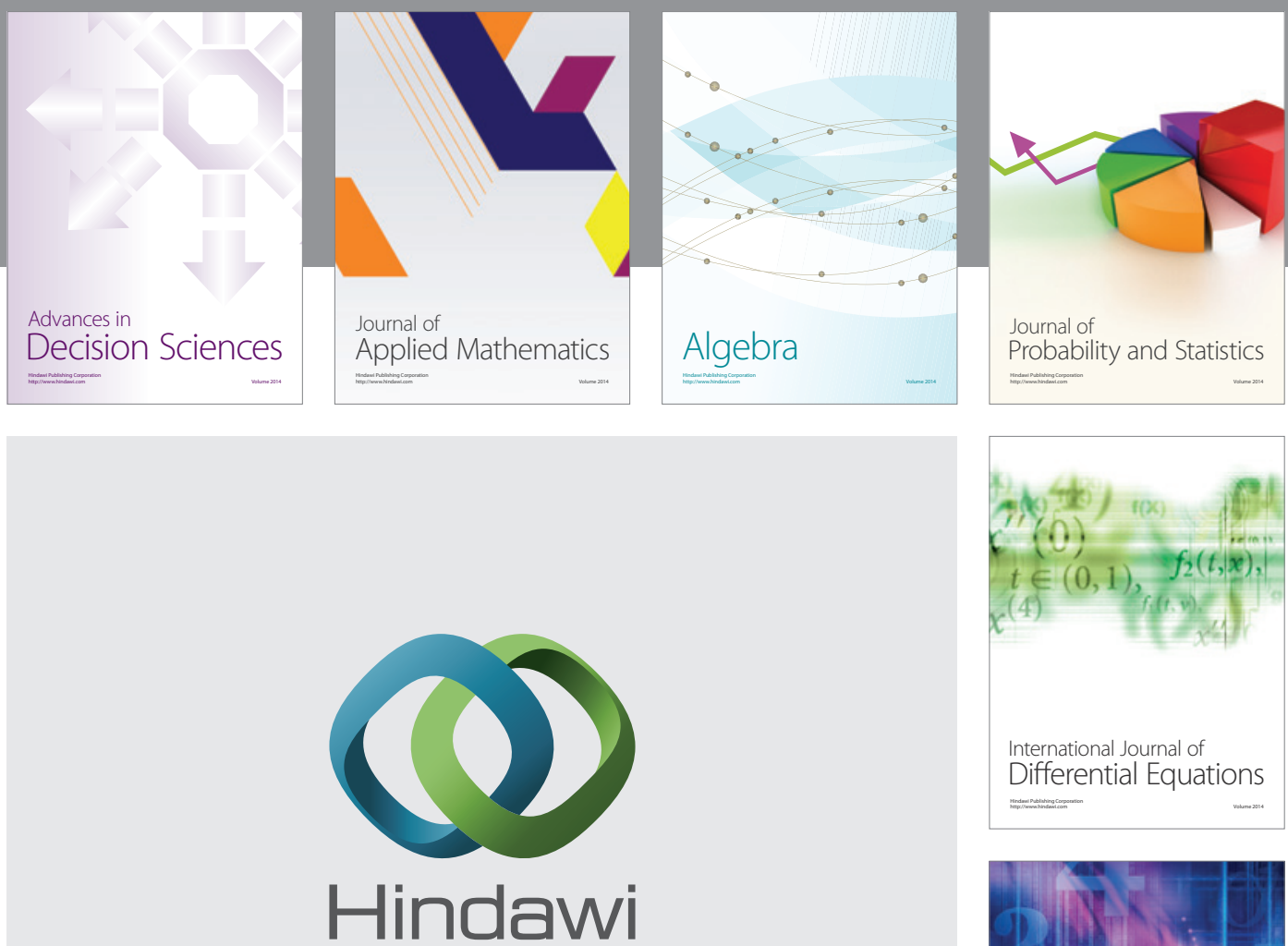

Submit your manuscripts at http://www.hindawi.com
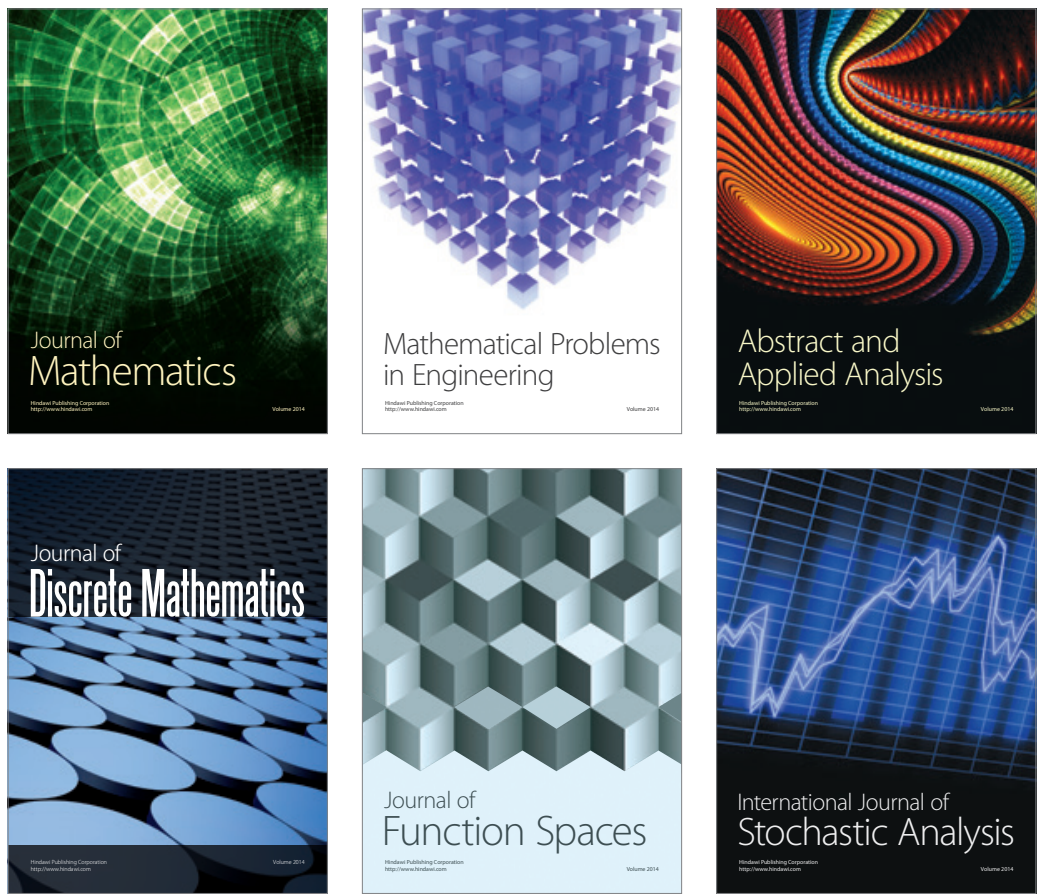

Journal of

Function Spaces

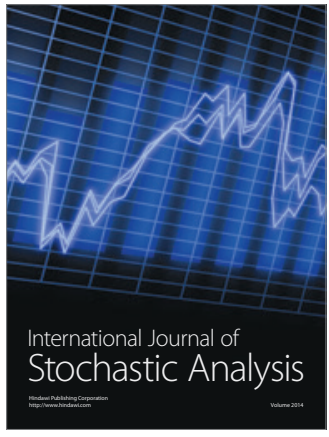

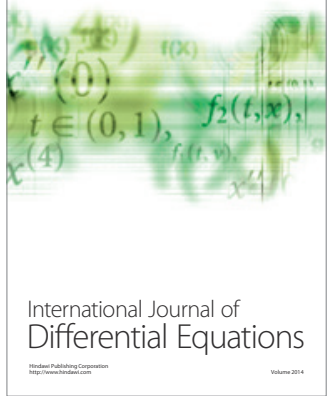
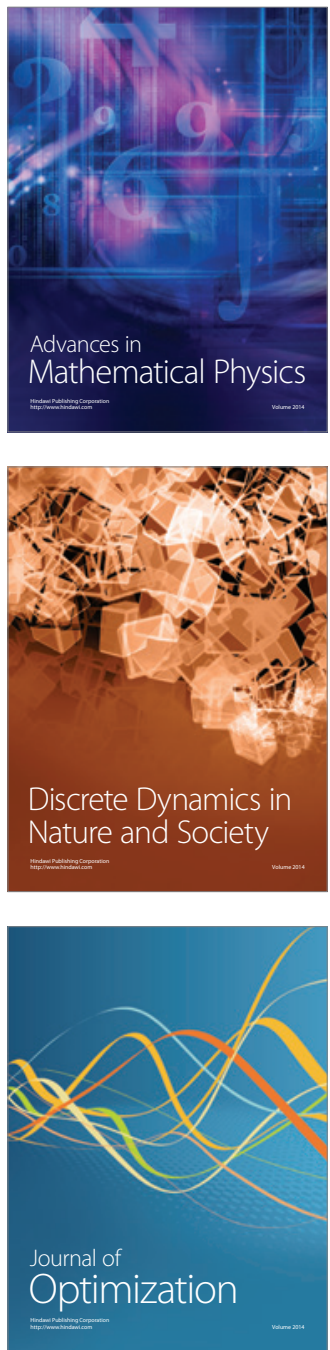